\title{
Nondestructive Measurement of Light-induced Oxidation in Dairy Products by Fluorescence Spectroscopy and Imaging
}

\author{
J. P. Wold,* K. Jørgensen,† and F. Lundby* \\ *MATFORSK Norwegian Food Research Institute, 1430 Ås, Norway \\ †TINE Norwegian Dairies BA, 4358 Kleppe, Norway
}

\begin{abstract}
The purpose of this paper is to demonstrate the potential of solid-sample fluorescence spectroscopy in nondestructive assessment of light-induced oxidation in different dairy products such as Swiss cheese, cream cheese, and sour cream. Analytical and quantitative spectral properties of fluorescence were elucidated by use of principal component analysis with designed experiments involving different levels of air and light exposure.
\end{abstract}

A significant reduction in fluorescence intensity at approximately $525 \mathrm{~nm}$, and a corresponding increase in the region 415 to $490 \mathrm{~nm}$ as a result of illumination was observed on all the products. The effect was ascribed to photodegradation of riboflavin. Variation in two smaller peaks at approximately $620 \mathrm{~nm}$ and 630 $\mathrm{nm}$ was an interaction effect between exposure to light and air. A pronounced interaction effect between light and air produced intense blue fluorescence and off-flavors on Swiss-like Jarlsberg cheese. High correlations (0.83 to 0.93 ) between fluorescence spectra and sensory measured off-flavors were obtained for cream cheese. Results indicate that solid-sample fluorescence can be used as a nondestructive and rapid tool to measure the degree of light-induced degradation of riboflavin as well as sensory properties connected to storage of dairy products. Images of fluorescence can be used to visualize the intensity and propagation of this process. The simplicity and rapidity of the method offer rich opportunities for efficient evaluation of factors affecting lightinduced oxidation in dairy products, such as packaging materials, light sources, exposure time, and temperature.

(Key words: light-induced oxidation, dairy product, fluorescence, image analysis)

Abbreviation key: ESR = electronic spin resonance, $\mathbf{P C}=$ principal component, $\mathbf{P C A}=\mathrm{PC}$ analysis, $\mathbf{P L S R}$

Received December 17, 2001.

Accepted January 30, 2002.

Corresponding author: J. P. Wold; e-mail: jens.petter.wold@ matforsk.no.
= partial least squares regression, $\mathbf{R M S E C V}=$ root mean square error of cross validation.

\section{INTRODUCTION}

Light is known to initiate oxidation processes resulting in discoloration and formation of off-flavors in foods (Bekbölet, 1990). Dairy products are often exposed to light during retail storage and display. This exposure can affect the quality of these products, especially when packed in transparent films or containers. Efforts are therefore being made to design protective packaging materials and nonharmful light sources. A problem is, however, that few or no methods for efficient and reliable evaluation of light-induced oxidation in dairy products are available. Kristensen et al. (2000) investigated various methods for Havarti cheese. They suggested two possible markers for light-induced oxidation: 1) free radicals measured by electronic spin resonance (ESR); and 2) the reduction of riboflavin as a result of photodegradation. Peroxide value correlated poorly with both sensory properties and storage time. It is generally accepted that riboflavin plays the role of photosensitizer in milk. The products of photosensitized oxidation are hydroperoxides that can decompose to make free radicals, which are responsible for autoxidation and the formation of off-flavors (Bekbölet, 1990). For cheeses and dairy spreads, however, the significance of riboflavin is not clear and seems to vary with the product depending on other factors such as the presence of antioxidants like $\beta$-carotene (Hansen and Skibsted, 2000; Li et al., 2000). ESR studies show that free radicals are indeed created in cheese at an early stage as a result of illumination (Kristensen and Skibsted, 1999). However, the light degradation of riboflavin in cheese did not correlate well with the ESR results nor with the sensory response to light (Deger and Ashoor, 1987; Kristensen et al., 2000). Motivated by this puzzle, we investigated the potential use of fluorescence spectroscopy to detect and measure photodegradation of dairy products.

Fluorescence is an intuitive choice for at least three reasons. First, the oxidation of unsaturated lipids in foods leads to the formation of hydroperoxides and secondary off-flavor compounds including aldehydes and 
ketones. These products can further react with macromolecules in the food matrix and create fluorescent compounds (Kikugawa and Beppu, 1987). These fluorescent compounds have successfully been utilized to detect lipid oxidation in foods by both solution and solid-sample fluorescence spectroscopy (Hasegawa, 1992; Aubourg, 1999; Wold and Mielnik, 2000). Liang (1999) showed that the correlation between storage time for milk powders and fluorescence $(R=0.95)$ was significantly higher than that between storage time and peroxide value or concentration of thiobarbituric reactive substances. Second, riboflavin is highly fluorescent, as are the breakdown products from photodegradation, lumiflavin, and lumichrome (Fox and Thayer, 1998). Since this process is believed to play a major role in the induction of free radicals and lipid oxidation, fluorescence can be expected to constitute an important marker of early lipid oxidation in dairy products. Third, solid-sample fluorescence spectroscopy is inexpensive, rapid, and nondestructive and lends itself to imaging techniques, such as the imaging of biochemical processes, e.g., lipid oxidation as proposed by Wold and Kvaal (2000).

Preliminary studies carried out at our laboratory showed pronounced changes in fluorescence from various dairy products after storage under different conditions of exposure to light and air. The purpose of this paper is to demonstrate the potential of solid sample fluorescence spectroscopy in nondestructive assessment of light-induced oxidation in different dairy products such as Swiss cheese, cream cheese, and sour cream. Analytical and quantitative spectral properties of fluorescence were elucidated by use of principal component analysis (PCA) with designed experiments involving different levels of air and light exposure. The relation between fluorescence and sensory assessed properties was analyzed by partial least squares regression (PLSR). We also illustrated how imaging of fluorescence can be used to visualize the intensity and spatial propagation of light-induced oxidation in the dairy products. Examples will be shown and possible applications discussed.

\section{MATERIALS AND METHODS}

\section{Materials}

Newly produced dairy products were collected at the Fellesmeieriet dairy plant in Oslo. The products were TINE goat cream cheese (Snøfrisk) (125-g boxes), light sour cream (3-dl cups), rindfree Jarlsberg cheese (Swiss-like) (400-g packages), and two loaves of cream cheese $(26 \times 8.5 \times 8.5 \mathrm{~cm}, 1.8 \mathrm{~kg}$ each $)$. The products were brought directly to the Institute and stored cool $\left(4^{\circ} \mathrm{C}\right)$ in the dark approximately $4 \mathrm{~d}$ before the experiments.

\section{Experimental Design}

Our first goal was to detect any changes in fluorescence caused by exposure to light and air. We did this by conducting exploratory designs using goat cream cheese, sour cream, and Jarlsberg cheese. Our second goal was to establish relationship between fluorescence and sensory attributes related to storage. This was achieved by conducting measurements on illuminated cream cheese.

Effects of light and air. To detect changes in fluorescence, we set up the following storage experiment:

All products were stored under four different conditions at $4^{\circ} \mathrm{C}$ : Light with exposure to air, light with no exposure to air, dark with exposure to air, and dark without exposure to air.

For each product we used two storage times and triplicate samples at each design point. This resulted in a total of 21 samples for each product $(4 \times 3 \times 2-3)$. Samples stored in the dark without air access would have been identical for the two storage times, so we used only one set, which explains the " -3 " above. All samples of each specific product were measured on the same day, meaning that they were introduced in the experiment at different times.

Illumination. A standard fluorescent tube (Osram L58W/21.840 Lumilux plus) was used for illuminating the samples during storage. It emits light mainly in the visible region, with some in the UV, and is commonly used for displays in grocery stores. The products were positioned $10 \mathrm{~cm}$ from the tube (which mimics conditions in grocery stores). Samples stored in the dark were placed in light-proof cases.

Goat cream cheese. Containers (125 g) of goat cream cheese were stored for 3 and $7 \mathrm{~d}$. Commercial triangular (sides $\approx 10 \mathrm{~cm}$ ) containers (height $\approx 2 \mathrm{~cm}$ ) of white plastic were used (material with high oxygen barrier (PP/EVOH/PP) from Forscheim, Germany). The boxes had a plastic white wafer seal (high barrier laminate (PETP/PE/EVOH/PE) from Ronsberg, Ronsberg, Germany) and a white plastic cover (PP/EVOH/PP), Forscheim, Germany). To allow air access, we removed the sealing, leaving those boxes with only the plastic cover. The boxes were illuminated from above, that is, the triangular surface under the cover was exposed to both air and light.

Sour cream. The sour cream cups were stored for 2 and $4 \mathrm{~d}$. Commercial 3-dl circular cups of white polystyrene (PLM, Malmö, Sweden) were used. The cups had a thin aluminum wafer seal and a circular polystyrene cover on top. To allow air access, we removed the alumi- 


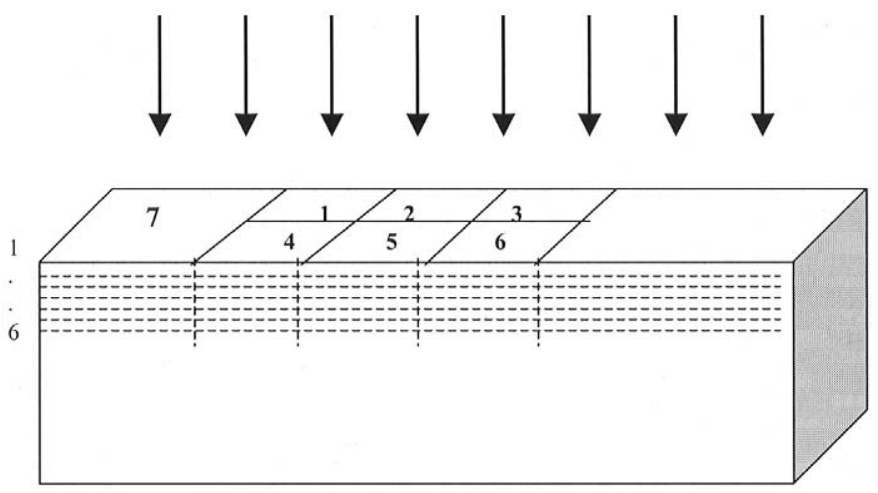

Figure 1. How the two cream cheeses were divided into subsamples at different layers. Subsamples 1 to 6 were used for sensory analysis, 7 was used for spectroscopic readings. Arrows indicate direction of illumination.

num wafer sealing from the top, leaving those cups with only polystyrene covers. Cups were illuminated from the side and above of a vertical standing fluorescent tube. That is, the horizontal top of the sample was exposed to both air and light, whereas the vertical side of the cup was exposed only to light.

Jarlsberg cheese. The cheese was stored for 3 and 7 $\mathrm{d}$ in commercial flow packages of transparent laminate plastic film (OPA 12/PE50 from Wipak, Nastola, Finland). The transparent side (without any labels or emblems) of the packages was turned against the light. Air access was achieved by puncturing the packages with a needle. That is, air had access to all sides, whereas only one side of the cheese was illuminated.

Riboflavin. As an additional control, a solution of $10 \mathrm{mg}$ of riboflavin (Sigma Chemical Co., St. Louis, MO) in $800 \mathrm{ml}$ of water was made. It was divided in two 400-ml portions, where one was stored in the dark, while the other was stored in the light under the same conditions as the dairy products.

Sensory assessment of illuminated cream cheese. Two whole loaves of cream cheese were illuminated for 4 and $7 \mathrm{~d}$ using the same fluorescent tubes as described above. They were vacuum packed in commercial transparent plastic film (Lakk/Cellofan/EVA from Danapak, Odense, Denmark). Sides without labels or emblems were turned toward the light. There was no air access (except for that allowed by the permeability of the packing material).

After storage, the loaves were divided into subsamples according to Figure 1. Six slices of thickness 2.5 $\mathrm{mm}$ were cut from the top of the loaf and down from the side that had been illuminated. Each slice was divided into six subsamples for sensory evaluation with the seventh subsample used for spectroscopic measurements. Every sensory judge tasted a total of 12 subsam- ples, six from each cheese. Fluorescence was measured on the corresponding subsamples. We had observed in advance that there were fluorescent gradients in the different products. The reason for evaluating different layers in the cheese was to determine whether the sensory panel was also able to detect and describe these fluorescent gradients in the cheese.

\section{Fluorescence Measurements}

The fluorescence emission spectra were measured directly on the cheese. An optical bench system optimized for measuring rather large sample surfaces (up to diam. $\approx 6 \mathrm{~cm}$ ) was used. The samples were illuminated by 380 $\mathrm{nm}$ excitation light, and fluorescence emission spectra were measured in the range 400 to $640 \mathrm{~nm}$. The excitation light was generated by a $300 \mathrm{~W}$ Xenon light source (Oriel 6258, Oriel Corporation, Stratford, CT) and a 10-nm bandwidth interference filter (Oriel 59920). The light was directed onto the samples at an angle of about $45^{\circ}$. The spectra were collected by an imaging spectrograph (Acton SP-150, Acton Research Corp., Acton, MA) connected to a sensitive charge coupled device (CCDcamera; Princeton TEA/CCD-512-TKBM1, Princeton Instruments Inc., Trenton, NJ). A cut-off filter at 400 $\mathrm{nm}$ (Melles Griot 03FCG049) was positioned in front of the spectrograph slit to suppress excitation light reflected from the sample. Exposure time was $1 \mathrm{~s}$ for all spectroscopic measurements. Temperature of samples was 16 to $19^{\circ} \mathrm{C}$. Two spectra were collected from each sample, and the average was used for further analysis. Spectrograph and detector were controlled by the software WinSpec Ver. 1.4.3.4 (Princeton Instruments Inc.). Spectra were digitized to a resolution of 16 bit and the length reduced from 512 to 128 points by averaging four adjacent readings, resulting in one reading for approximately every second nanometer.

Images were collected with the same system, slightly modified. A Nikon $102 \mathrm{~mm}$ photographic lens was mounted on the imaging spectrograph, the spectrograph slit was removed and the grating exchanged with a mirror. Spectral images were created by placing 40 $\mathrm{nm}$ bandwidth interference filters in front of the lens. Samples were illuminated by $380 \mathrm{~nm}$ light and images were taken at 450 and $550 \mathrm{~nm}$. Exposure time for all images was $15 \mathrm{~s}$.

Goat cream cheese. Fluorescence spectra were measured on all 21 samples. The following measurements were performed on each sample: 1) plastic cover and plastic wafer were removed from the container and fluorescence was measured on the surface of the cheese while in the container (measured circular area: $\mathrm{D} \approx 5$ $\mathrm{cm})$; 2) The cheese was tipped out of the container and a vertical cut was made through the middle. One of the 
rectangular cross sections was measured $(\mathrm{A} \approx 2 \times 5 \mathrm{~cm})$; and 3 ) the other cross section was imaged. The purpose was to determine whether it was possible to detect an fluorescence gradient in the image.

Sour cream. Fluorescence spectra were measured on 14 of 21 samples (two of three triplicates). The following measurements were performed: 1) plastic cover and aluminum wafer were removed from the cups and fluorescence was measured on the surface (measured circular area: $\mathrm{D} \approx 5 \mathrm{~cm}$ ); 2 ) sour cream against the vertical side of the cup exposed only to light was drawn out with a spoon, placed in cuvettes $(\mathrm{D}=5 \mathrm{~cm})$ and measured; and 3 ) The remaining seven samples were frozen $\left(-25^{\circ} \mathrm{C}\right)$ and thawed 2 wk later for imaging. Each cup was cut vertically in half with a sharp knife along the side that had been exposed to light. The cross sections were then imaged.

Jarlsberg cheese. Fluorescence spectra were measured on 14 of 21 samples (two of three triplicates). 1) A cylinder $(\mathrm{D}=5 \mathrm{~cm})$ was cut from the center of each cheese. The flat top of the cylinder was the illuminated surface of the cheese. This top surface was measured. Then, with a cheese slicer, we sectioned 1-mm thick slices off the cylinder from the top. For each slice, a new measurement was done in order to investigate possible fluorescence gradients in the samples; 2) A cross section of each of the last seven samples was imaged.

Cream cheese. Fluorescence spectra from the 12 subsamples (six from each loaf) that had been subjected to sensory evaluation were all measured at site no. 7 (Figure 1).

Riboflavin. Fluorescence spectra were measured from the two riboflavin solutions.

\section{Sensory Evaluation}

The sensory evaluation was performed by a trained sensory panel with special skills for evaluating dairy products. The panel, consisting of six trained assessors, carried out a prescribed descriptive test (ISO 1985). Before the analysis, the panel was trained in the definition and intensities of the four chosen attributes using cream cheese with varying sensory properties. These attributes were oxidized odor, oxidized flavor, total odor, and total flavor. Each assessor was served each cheese sample on a cardboard plate. The judgement was performed in two rounds, one for each of the two cream cheese loaves. The serving order was randomized according to sample and assessor. Water and crackers were provided to cleanse the palate between samples. A continuous nonstructured scale was used for evaluation of sensory attributes ranging from the lowest intensity of each attribute (value 1.0) to the highest intensity (value 9.0). Each judge evaluated the samples at indi- vidual speed on a computer system for direct recording of data (CSA, Compusense, Version 4.2, Guelph, ON, Canada). The sensory scores for each layer of cheese were obtained by averaging the individual scores for the six subsamples from the same layer.

\section{Data Analysis}

PCA was applied to the spectral data from all the experiments in order to get the best possible view of the spectral structure. Scores, loadings, and explained variance were studied for the first three to four principal components (PC). Loading plots were used to interpret the spectral variation contained in each $\mathrm{PC}$, while score plots were used to visualize the relation between samples in the corresponding PC. The spectra were not subjected to any kind of preprocessing before analysis.

The PLSR (Martens and Næs, 1989) was attempted for making a calibration between fluorescence and sensory assessed attributes on cream cheese. Full cross validation was used for all models. This provided a predicted value for each sample, $\hat{y}_{i}$, which was compared to the reference sensory value, $y_{i}$. Multivariate correlation coefficient $(R)$ and the prediction error expressed as root mean square error of cross validation (RMSECV) were used to evaluate the models. RMSECV was defined as

$$
R M S E C V=\sqrt{\frac{1}{N} \sum_{i=1}^{N}\left(y_{i}-\hat{y}_{i}\right)^{2}}
$$

where $i$ denotes the samples from 1 to $N$. Data analyses were performed with the software The Unscrambler version 7.5 (Camo AS, Oslo, Norway).

Images were imported into ImagePro Plus version 4.5 (Media Cybernetics, L.P., Silver Spring, MD). Possible gradients in fluorescence intensity were detected by simply studying the gray level values of the pixels.

\section{RESULTS AND DISCUSSION}

Results for each product will be presented separately. Common to all products was a powerful fluorescence easily seen by the naked eye. The apparent trend was that illuminated areas of the products exhibited a violet fluorescence, while nonilluminated areas showed a yellowish/white fluorescence.

\section{Goat Cream Cheese and Sour Cream}

Figure $2 \mathrm{a}$ and $\mathrm{b}$ show the main spectral features of the fluorescence from goat cheese and sour cream measured at the surface (measurement no. 1), respectively. 

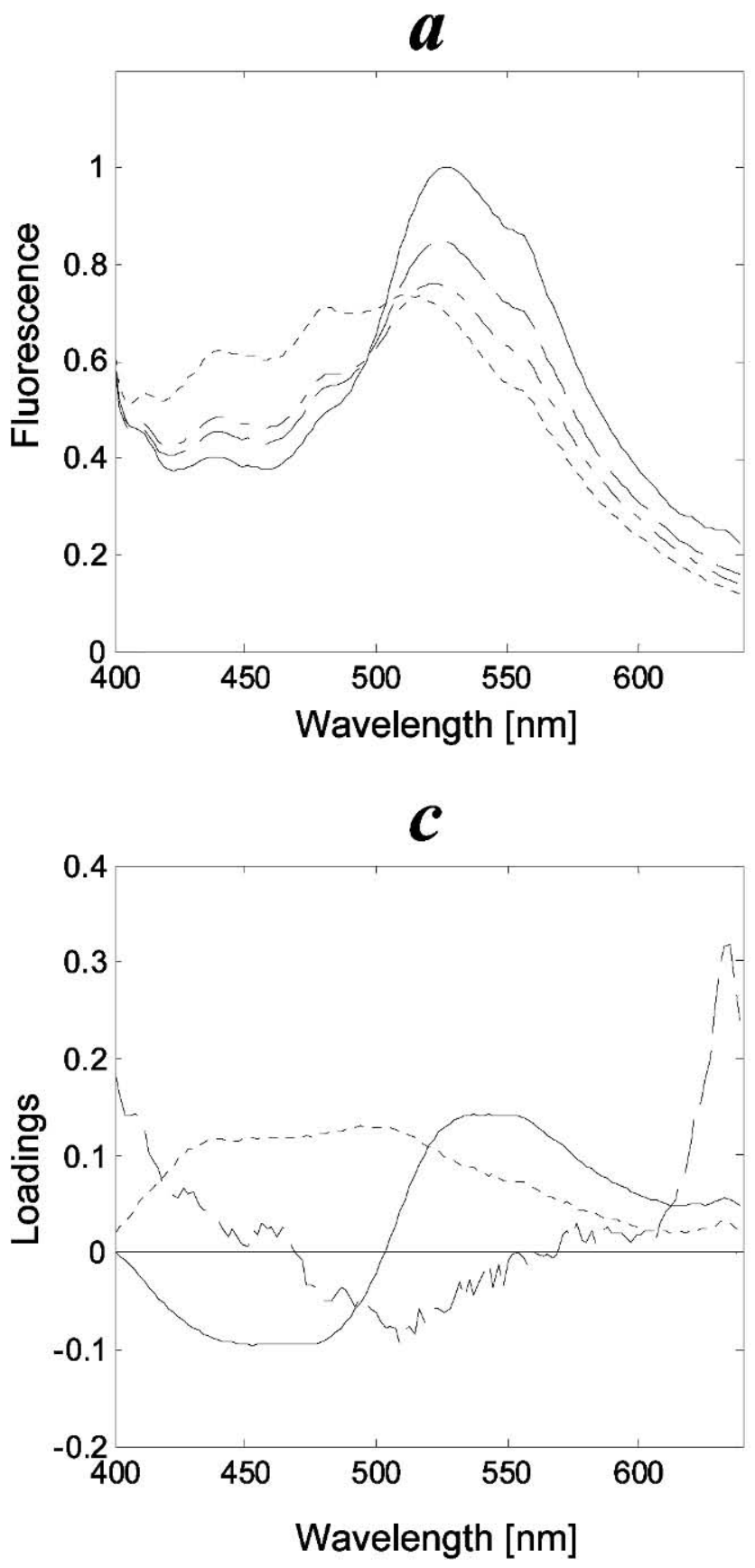
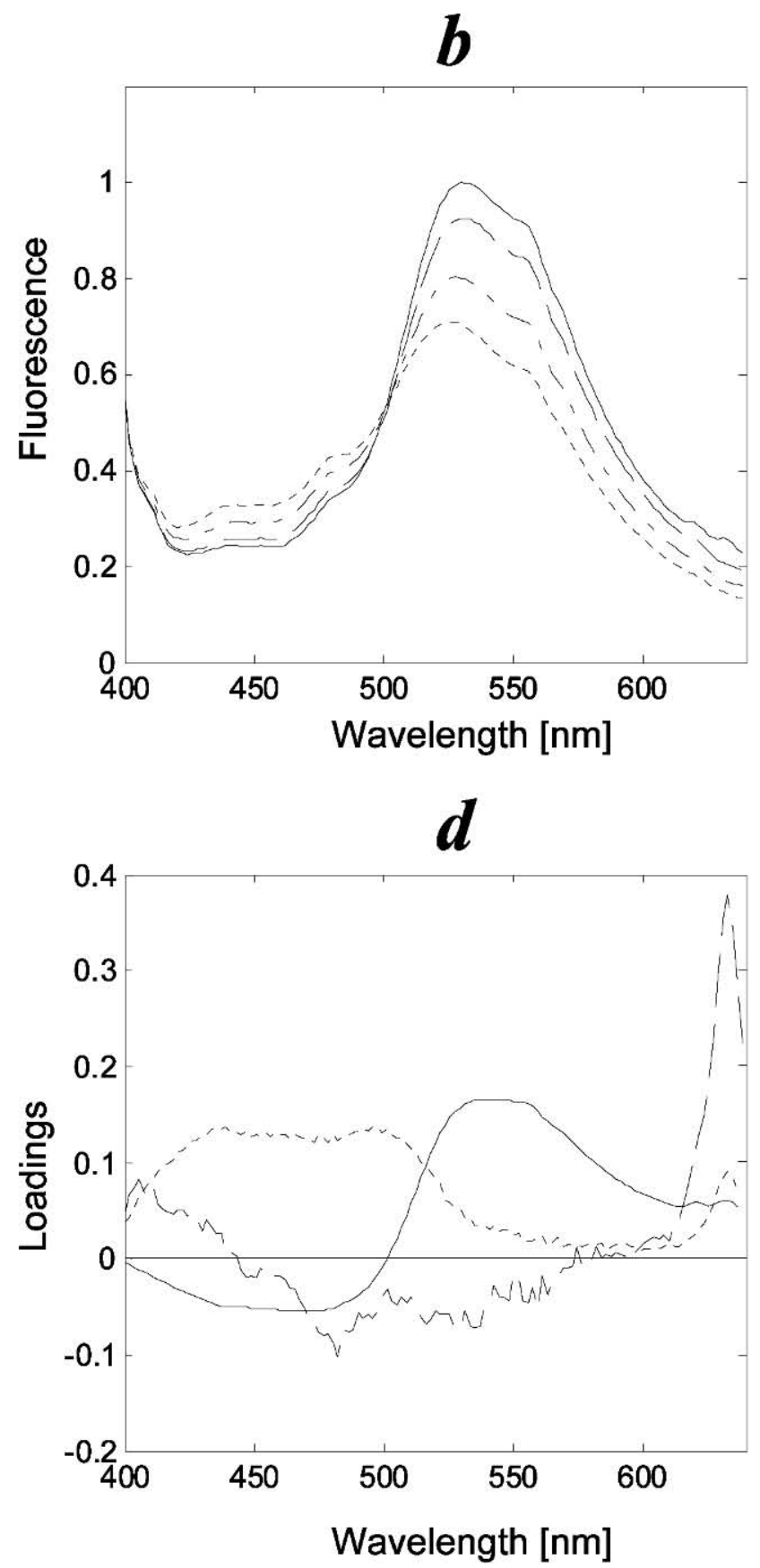

Figure 2. Fluorescence spectra from goat cream cheese (a) and sour cream (b). Nonilluminated samples: —, $3 / 2 \mathrm{~d}$ storage, illuminated without air:,$---- 3 / 2 \mathrm{~d}$ storage, illuminated with air: $-\cdot-\cdot-\cdot, 7 / 4 \mathrm{~d}$ storage, illuminated without air: -------. Loadings for the first three principal components of principal component analysis of fluorescence measurements on goat cream cheese (c) and sour cream (d). PC1: — , PC2: --------, PC3: ----.

There are two spectral regions that are of particular interest; the broad peak around 520 to $530 \mathrm{~nm}$ (designated the $525 \mathrm{~nm}$ peak) which is yellow/green in color, and the region 415 to $490 \mathrm{~nm}$, which is violet and blue. Visually, it is apparent that samples stored in the dark demonstrated intense fluorescence around $525 \mathrm{~nm}$, and correspondingly low fluorescence in the violet region. Illuminated samples, however, showed less difference between these regions, indicating a reduction of fluorescence around $525 \mathrm{~nm}$ and an increase in the violet area. Consequently, illuminated samples appeared more violet. It was also observed that this change was 


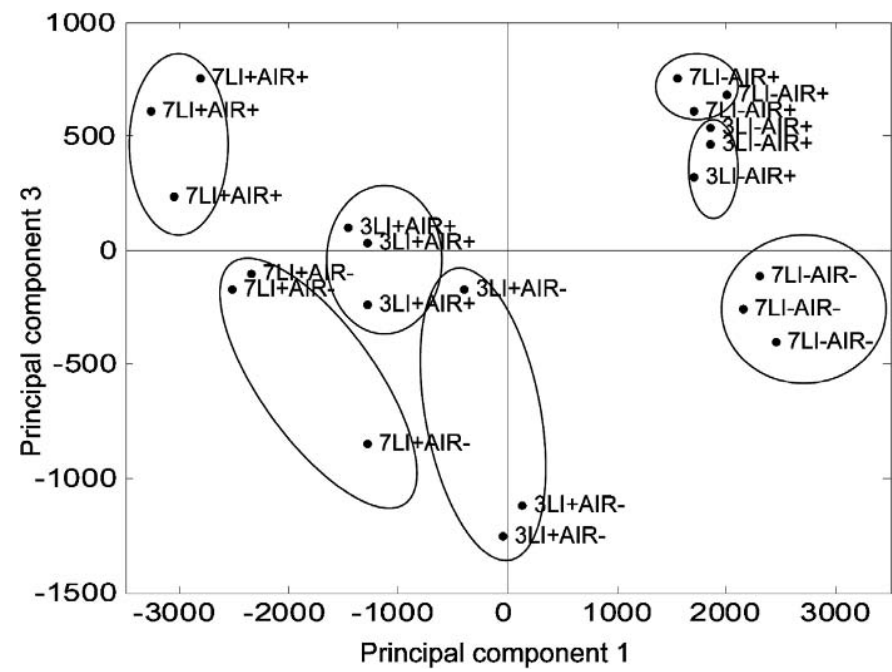

Figure 3. Scores for principal components 1 versus 3 for fluorescence measurements on goat cream cheese. Sample names are described by storage time ( 3 or $7 \mathrm{~d}$ ), with or without illumination (LI+ or LI-), and with or without air access (AIR+ or AIR-).

gradual and seemed to have been mostly dependent on light exposure time. It is noteworthy that the fluorescent properties for the two products are very similar, differing only by a more intense fluorescence in the 415 to $490 \mathrm{~nm}$ for the longest illuminated goat cheese. This might be explained by the longer storage period (7 vs. $4 \mathrm{~d}$ for sour cream). The loading plots shown in Figure $2 \mathrm{c}$ and $\mathrm{d}$ confirm the spectral similarities between the two products. The spectral shapes of the three first principal components were almost identical. First component explained 96.7 and $98.1 \%$ of the spectral variation in goat cheese and sour cream, respectively. This component represents the reduction at $525 \mathrm{~nm}$, and some of the increase at 415 to $490 \mathrm{~nm}$. Second component (3.1 and $1.5 \%$ explained variance, respectively) expressed additional variation in the 415 to $540 \mathrm{~nm}$ region but was not correlated with the reduction at 525 $\mathrm{nm}$. Third component $(0.18$ and $0.26 \%)$ had most of its variation in a narrow spectral region at approximately $630 \mathrm{~nm}$. This peak corresponds to the two very small bumps in the raw spectra at 620 and $630 \mathrm{~nm}$.

The design parameters causing these spectral effects can be fairly well visualized by the score plot of PC1 versus PC3 (Figure 3). The score plot is shown only for the goat cheese data, which were very similar to that of sour cream. Samples in the plot were named according to the design parameters and show that the triplicate samples are well clustered. It can be seen that PC1 explains variation caused by the degree of light exposure. Samples exposed for $7 \mathrm{~d}$ are all to the left, whereas those stored in the dark cluster on the right side in the plot. Illuminated samples that had access to air lie slightly more to the left in the plot compared with those that were not exposed, probably because the plastic wafer under the cover was removed, allowing more light to reach the cheese. This effect was also observed for the sour cream but was much more pronounced since the aluminum wafer was a more efficient blocker of light. PC3 seemed to separate samples exposed to air from those that were not. Samples with air had positive score values, whereas those without air had negative values. The explanation of PC3 is supported by the measurements performed on sour cream located against the vertical side of the cup (measurement no. 2). This material was not exposed to air, only to light coming through the cup. The PCA on these data showed only the two first major components, but the third component with the loading peak at $630 \mathrm{~nm}$ was not present. It should be mentioned that the scores of PC2, not shown, also gave clear clusters of similarly treated samples, but the values did not relate to any of the design parameters.

Measurements performed on the cross sections of the goat cheese (no. 2) showed the same spectral features as those measured at the top surface of the cheese, although the variation in intensity was much less. This indicated that the violet fluorophores measured on the surface also could be detected in the interior of the cheese. This was verified by the fluorescence images (Figure 4) comparing two cross sections, one cheese stored in the dark and one in the light for $7 \mathrm{~d}$. Since the images were taken with a broad band filter around $550 \mathrm{~nm}$, the reduction of the peak at $525 \mathrm{~nm}$ lead to a darker region from the top of the illuminated cheese. The image also shows that this darker region penetrated approximately half way into the cheese $(\sim 7$ to 8 $\mathrm{mm}$ ), suggesting that the effect of light exposure propagated a considerable distance into the cheese. The images of sour cream cross sections (Figure 5) show a similar trend. These images were taken at $450 \mathrm{~nm}$, consequently presenting the strong violet fluorescent areas as the brightest. Again, it can be seen that the illumination created a change in fluorescence which penetrated 4 to $5 \mathrm{~mm}$ into the material.

These images are interesting in many ways. They show us both the intensity and propagation of an effect caused by illumination. Whatever reaction is responsible for the change in fluorescent properties, it can be used as a marker for light penetration in dairy products. The significance of this fluorescent gradient to cheese quality will be elucidated by the cream cheese experiment discussed below.

Even without any chemical reference analyses, it is possible to suggest possible reactions leading to the changes in fluorescence. Riboflavin has a strong and broad fluorescence emission peak in the region 525 to 

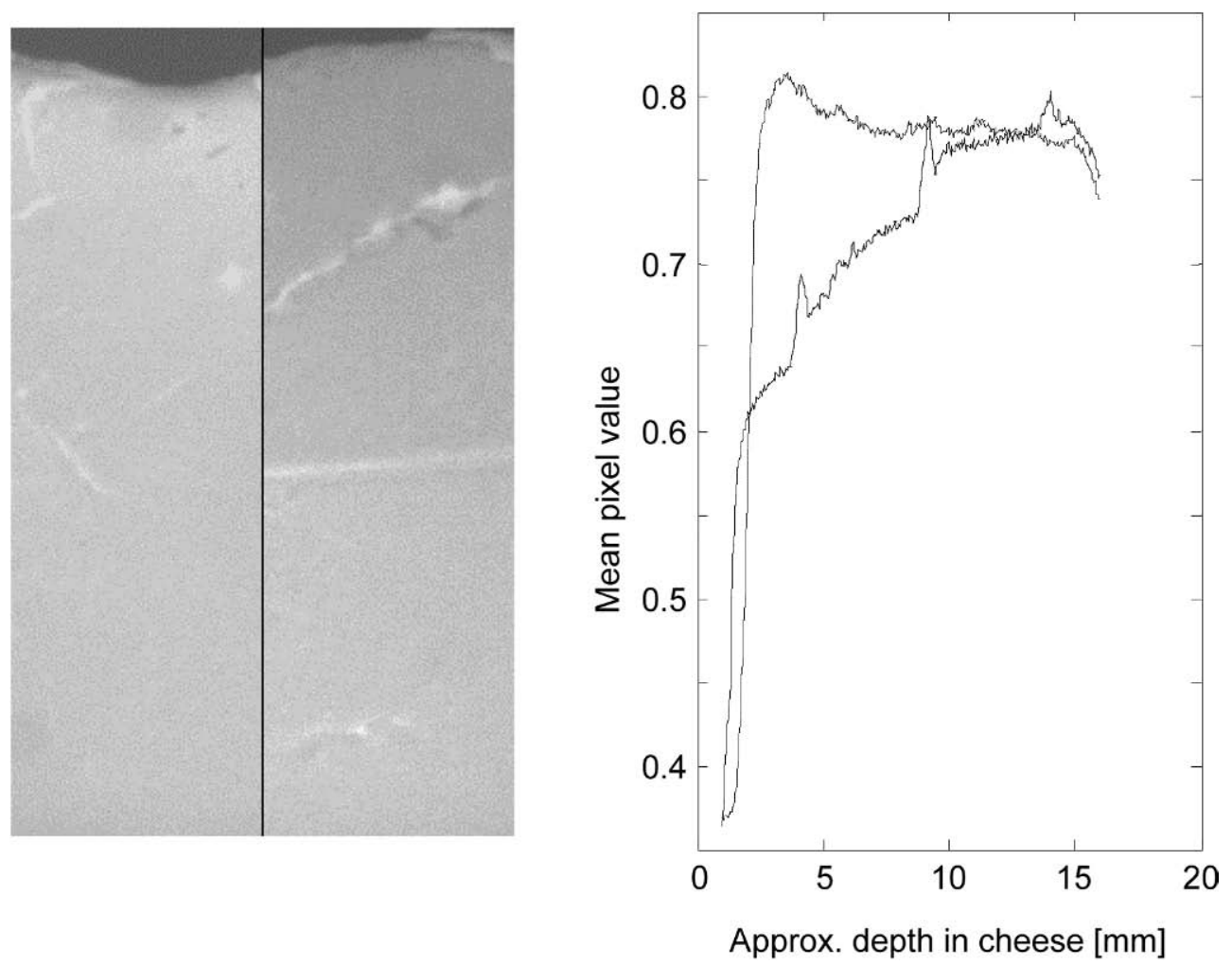

Figure 4. Image of two cross sections of goat cream cheese. The right cheese was illuminated from above for $7 \mathrm{~d}$. Note the dark region penetrating from the top and into about the middle of the cheese. Left cheese was stored in dark. The bright stripes and grooves are small damages in the cheese surface, and is not of relevance. The graph shows the mean pixel values across the image from the top and downwards in the cheese. High values correspond to bright pixels and visa versa. Light exposed cheese: — - , cheese stored in the dark:

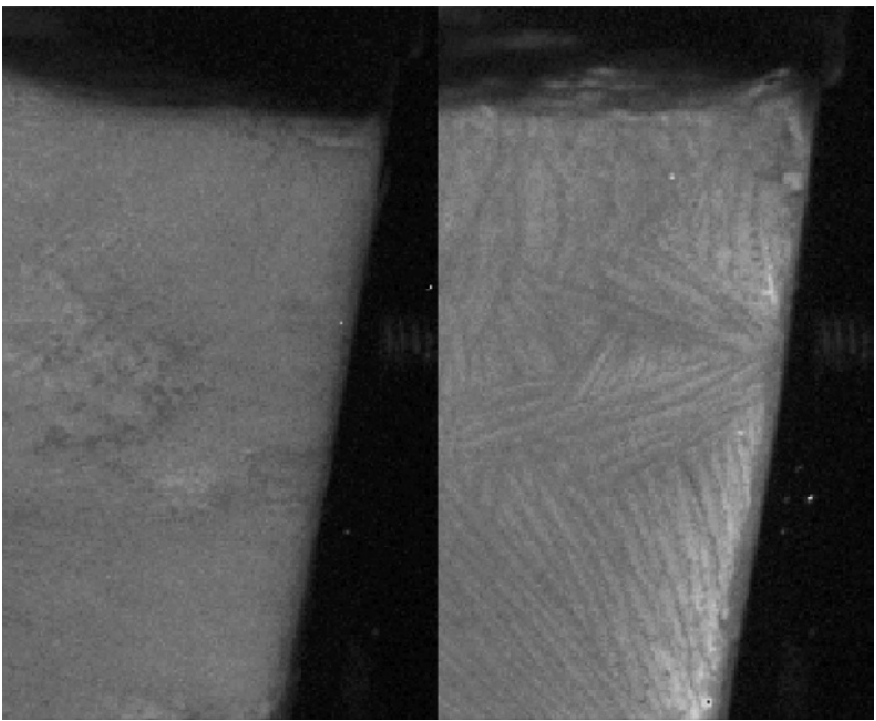

Figure 5. Image of two cross sections of sour cream. The cup to the right was illuminated from the right for $4 \mathrm{~d}$. Note the bright region stretching from the side and into the sour cream. Left cup of sour cream was stored in dark. The criss-crossing stripes are crystals due to frozen storage of the sour cream, and are not of relevance. Height of the cups is $8 \mathrm{~cm}$.
$531 \mathrm{~nm}$ (Fox and Thayer, 1998). It also has a broad excitation spectrum including $380 \mathrm{~nm}$, the excitation wavelength used in our study. It is known that when riboflavin is illuminated by fluorescent light, it is photochemically degraded into different forms of lumichrome and lumiflavin. These compounds are also fluorescent with emission maxima in the regions 444 to $479 \mathrm{~nm}$ and 516 to $522 \mathrm{~nm}$, respectively (Fox and Thayer, 1998). Furthermore, lumichrome has been found to occur naturally in milk and pasta exposed to light (Parks and Allen, 1977; Woodcock et al., 1982; Toyosaki and Hayashi, 1993). Although the reported excitation and emission maxima were found by solution spectroscopy, the mechanism seems to fit our observations for cheese and sour cream. The reduction of fluorescence around 525 $\mathrm{nm}$ might reflect the degradation of riboflavin. Some of the increase in the region 415 to $490 \mathrm{~nm}$ might be caused by the formation of lumichrome. There was also a slight shift of the $525 \mathrm{~nm}$ peak towards $520 \mathrm{~nm}$ (especially for the goat cheese) which might involve the formation of lumiflavin. Fluorescence spectrophotometers might produce fluorescence spectra with slight differences because they consist of different optical components. To make a proper comparison between fluores- 


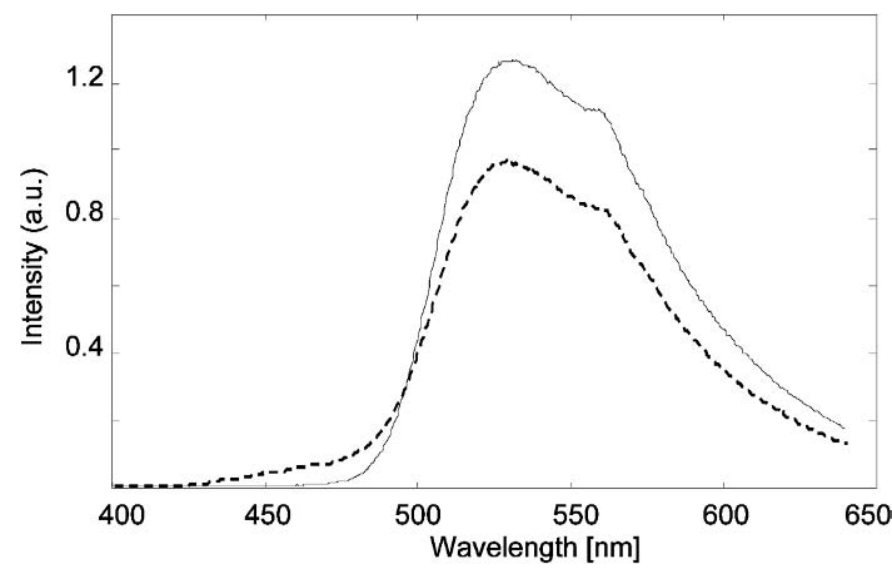

Figure 6. Fluorescence spectra of riboflavin solutions in water. Solution stored in dark: ——, illuminated solution: ---------- .

cence from the dairy products and riboflavin, it was important to measure the riboflavin solutions with our system. Figure 6 shows spectra from the riboflavin solution stored in the dark as well as from the same solution illuminated for $3 \mathrm{~d}$ with the same lamp that was used on the dairy products. The solution had an emission peak at about $525 \mathrm{~nm}$. The illuminated solution exhibited significantly less fluorescence around $525 \mathrm{~nm}$, but an increase in the region 420 to $510 \mathrm{~nm}$, probably caused by formation of lumichrome. Note that the spectra for riboflavin are very similar to the major peaks obtained for sour cream and goat cheese. This experiment supports the hypothesis that photochemical degradation of riboflavin caused the major change in fluorescence.

Photodegradation of riboflavin in cheese has not been widely studied. A reduction of riboflavin in Cheddar cheese after $12 \mathrm{~d}$ of storage was found to be in the range 24 to $44 \%$, depending on the light intensity (Deger and Ashoor, 1987). Kristensen et al. (2000) studied lightinduced oxidation in sliced Havarti cheese and found a significant decrease in riboflavin with light exposure, first appearing after $11 \mathrm{~d}$ of storage. In this study, we observed obvious effects on sour cream after $2 \mathrm{~d}$ and goat cheese after $3 \mathrm{~d}$. Photodegradation of riboflavin in milk is well elucidated (Bekbölet, 1990). It is dependent on the wavelength and intensity of light, exposure time, the protective effect of the packing material, and on temperature. Allen and Parks (1979) found that the degradation followed a first-order reaction rate, that is, it started immediately at illumination. The same conclusion was drawn for pasta, where a rapid initial phase followed by a slower degradation phase was observed (Woodcock et al., 1982). Allen and Parks (1979) also concluded that the photodegradation of riboflavin occurred before the appearance of the light-induced off- flavors. This has later been verified through many studies, advocating that riboflavin plays the role of a photosensitizer. The singlet state sensitizer is converted to an exited triplet sensitizer by light. The exited triplet sensitizer can react with food components such as protein or fat to produce free radicals and radical ions. The exited triplet sensitizer can also react with atmospheric triplet oxygen creating singlet oxygen. The highly unstable singlet oxygen can directly react with electronrich compounds such as double bonds in fatty acids and fat-soluble vitamins to produce undesirable flavor compounds (Aurand et al. 1976; Li et al., 2000).

The variation in the region 410 to $560 \mathrm{~nm}$, which did not correlate with the reduction of the peak at 525 $\mathrm{nm}$ (PC2), might be ascribed to the reaction between primary and secondary lipid oxidation products with proteins to form fluorescent compounds (Kikugawa and Beppu, 1987). Such products typically exhibit fluorescence between 400 and $500 \mathrm{~nm}$. For example, Liang (1999) found that oxidized milk powder has an emission maximum at $450 \mathrm{~nm}$ for solid-sample fluorescence.

Lastly, the effect of the third PC was, as mentioned, probably caused by exposure to air. So far, we have not elucidated the chemical origin of the two tiny peaks at 620 and $630 \mathrm{~nm}$.

\section{Jarlsberg Cheese}

Fluorescence measurements on Jarlsberg cheese revealed many of the same properties observed for goat cheese and sour cream. One significant difference was, however, a very powerful blue fluorescence emitted from the surface of cheeses that had been exposed to both light and air. These samples were also accompanied by intense off-flavors and suffered from discoloration (less yellow, more pale). Figure 7 shows spectra measured on the six uppermost cheese slices taken from a sample stored for $7 \mathrm{~d}$ in light with access to air. The first slice at the surface (slice 1) exhibited a strong blue fluorescence peaking at approximately $480 \mathrm{~nm}$; however, at slice 2 we observed a spectrum similar to those observed on the other dairy products. Penetrating deeper into the cheese the intensity at $525 \mathrm{~nm}$ continued to increase until stabilizing at 5 to $6 \mathrm{~mm}$ depth (slice no. 5 and 6). A spectrum from a surface slice of a cheese stored in the dark is plotted for comparison. The strong blue fluorescence was observed down to a depth of about $1 \mathrm{~mm}$. The images in Figure 8 illustrate these observations. The two upper images are of the same cheese sample, the topmost taken at $450 \mathrm{~nm}$ and the other one at $550 \mathrm{~nm}$. The topmost slice showed the propagation of the blue fluorescence as a bright zone, whereas the middle image showed the reduced intensity at $525 \mathrm{~nm}$ as a dark region stretching about $6 \mathrm{~mm}$ into 


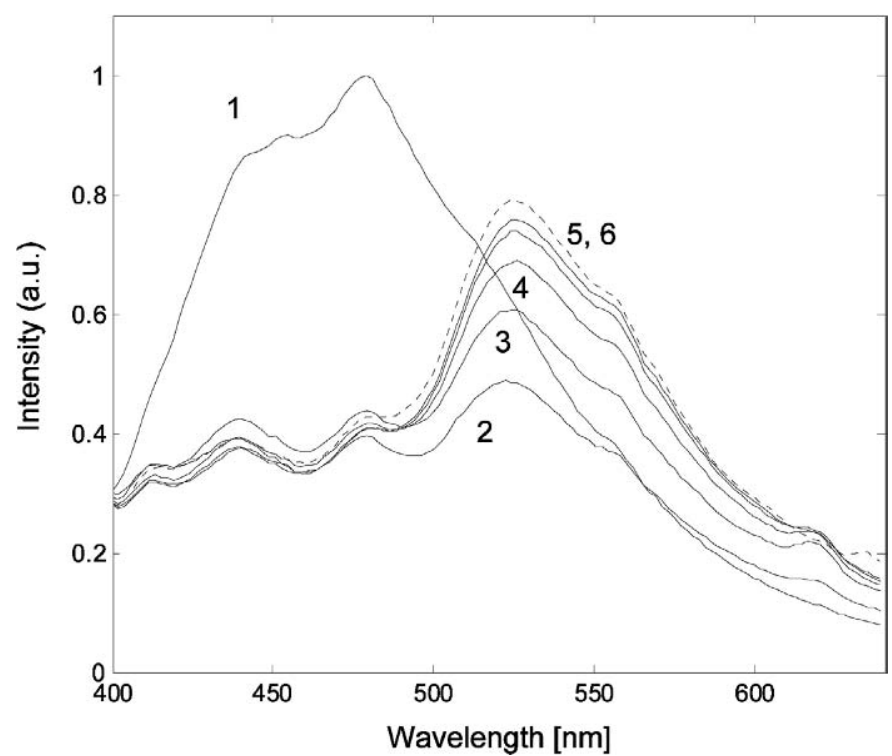

Figure 7. Fluorescence spectra from Jarlsberg cheese exposed to light and air for $7 \mathrm{~d}$. Numbers indicate slice number from the illuminated surface and downwards in the cheese. Slices were about $1 \mathrm{~mm}$ thick. The dotted spectrum is from the surface of a cheese stored in the dark without air access.

the cheese, in accordance with the spectral results in Figure 7. The lower cheese was stored in the dark and showed no obvious gradients in fluorescence.

The bright blue surface fluorescence differed markedly from what we observed on goat cheese and sour cream. However, when these surface samples were omitted from the dataset, a PCA revealed spectral properties similar to those shown in Figure 2c and d, indicating that exactly the same reaction mechanisms were present in Jarlsberg cheese. The very intense interaction effect between light and air leading to discoloration and off-flavors might be a result of singlet oxygen reactions. A similar observation was made by Hong et al. (1995) who noticed loss of yellowness for illuminated Cheddar cheese, especially when packaging film with high oxygen transmission was used.

\section{Fluorescence and Sensory Analysis of Cream Cheese}

The sensory evaluation was carried out in an effort to establish a connection between fluorescence and quality properties related to storage. During the above-discussed experiments, we generally noticed off-flavors connected to oxidation from the illuminated samples. In the sensory evaluation, we focused on the effect at $525 \mathrm{~nm}$, which was observed in all the dairy products. The experimental design also allowed us to investigate whether it was possible to correlate sensory description with the observed gradients in fluorescence.

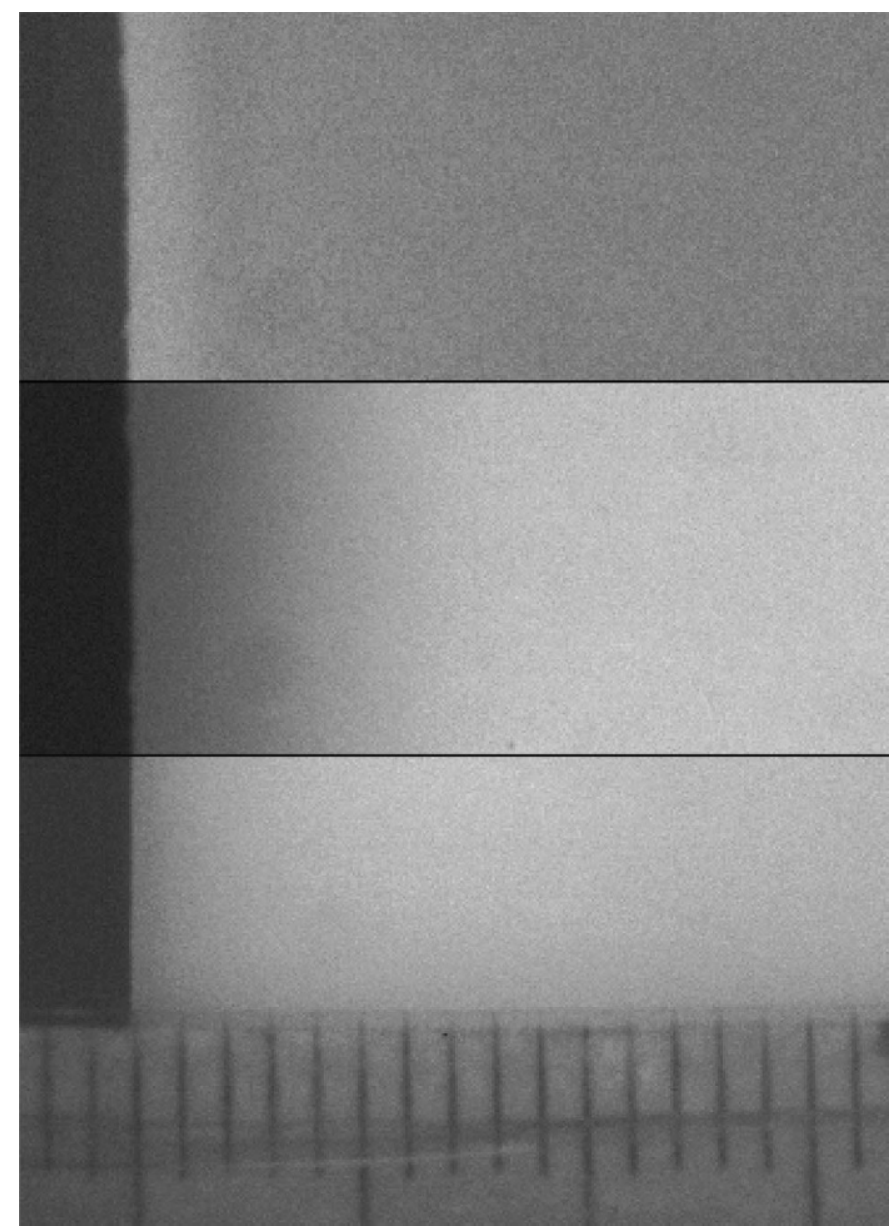

Figure 8. Three images of fluorescence from cross sections of stored Jarlsberg cheese. Upper image taken at $450 \mathrm{~nm}$ : Jarlsberg cheese exposed to light and air for $7 \mathrm{~d}$. Middle image taken at 550 $\mathrm{nm}$ : Jarlsberg cheese exposed to light and air for $7 \mathrm{~d}$. Lower image taken at $550 \mathrm{~nm}$ : Jarlsberg cheese stored dark and without air access. A ruler indicates $\mathrm{mm}$.

The sensory panel could indeed register a gradient in oxidized odor and flavor for both cream cheeses. Figure 9 shows the mean sensory scores for these properties moving inwards in the cheese. The results indicate that light-induced oxidation propagated somewhat further into the 7-d cheese compared with the 4-d cheese, and that the sensory scores were also higher until reaching a minimum at approximately $7.5 \mathrm{~mm}$. From 7.5 to $12.5 \mathrm{~mm}$, the sensory scores leveled out, indicating that the cheese at this point and deeper was "fresh." Standard deviation for each measurement is not included in the curves, as the variation between judges was relatively large. For both oxidized flavor and odor, there was no significant difference between the three upper layers ( 0 to $5 \mathrm{~mm}$ ); however, these layers had significantly higher scores compared with the three 
a

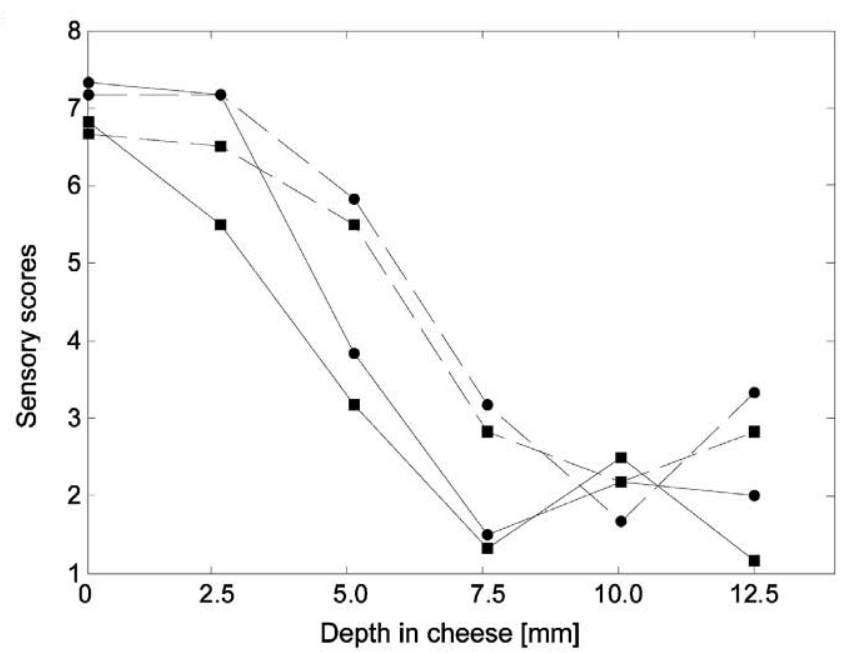

b

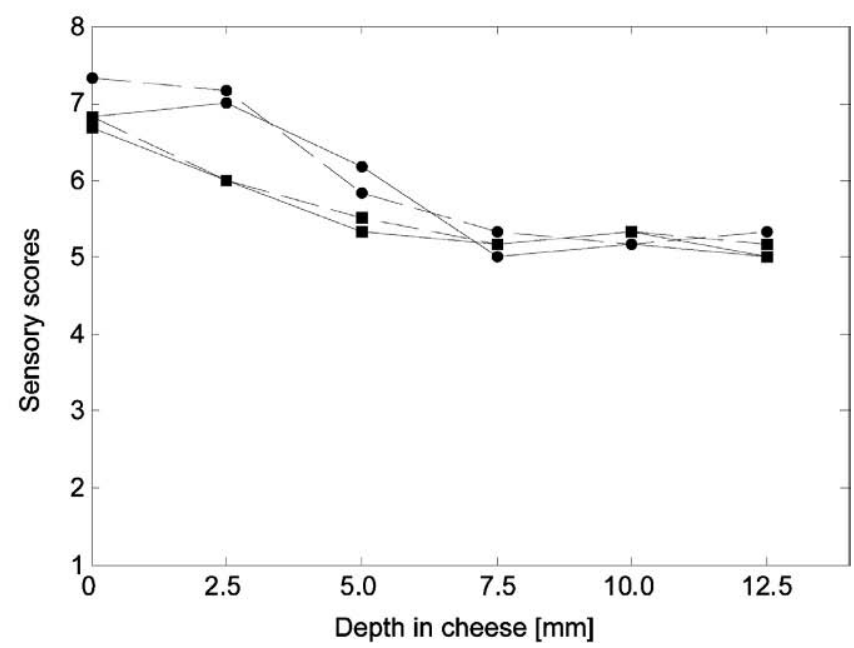

Figure 9. Sensory scores for the two illuminated cream cheeses as function of depth. a) Oxidized odor ( $)$ and oxidized flavor $(\mathbf{)}$ in 7-d cheese (-------) and 4-d cheese (-). b) Total odor (ם) and total flavor (O) in 7-d cheese (-------) and 4-d cheese (-).

lower layers (7.5 to $12.5 \mathrm{~mm}$ ). The same results applied for the attributes of total odor and total flavor.

The fluorescence spectra from cream cheese resembled those measured on the other dairy products (Figure 10). There was an obvious variation at the $525 \mathrm{~nm}$ peak as a function of depth. The uppermost layer displayed low intensity in this region, which increased inwards in the cheese. Note that the three innermost layers had similar spectra, indicating the end of the fluorescent gradient. A close relation between fluorescence intensity and sensory scores at the different layers is apparent. Note also how the peaks at 620 and $630 \mathrm{~nm}$ vary as a function of depth.

The regression results emphasize the strong correlation between fluorescence and sensory properties (Table

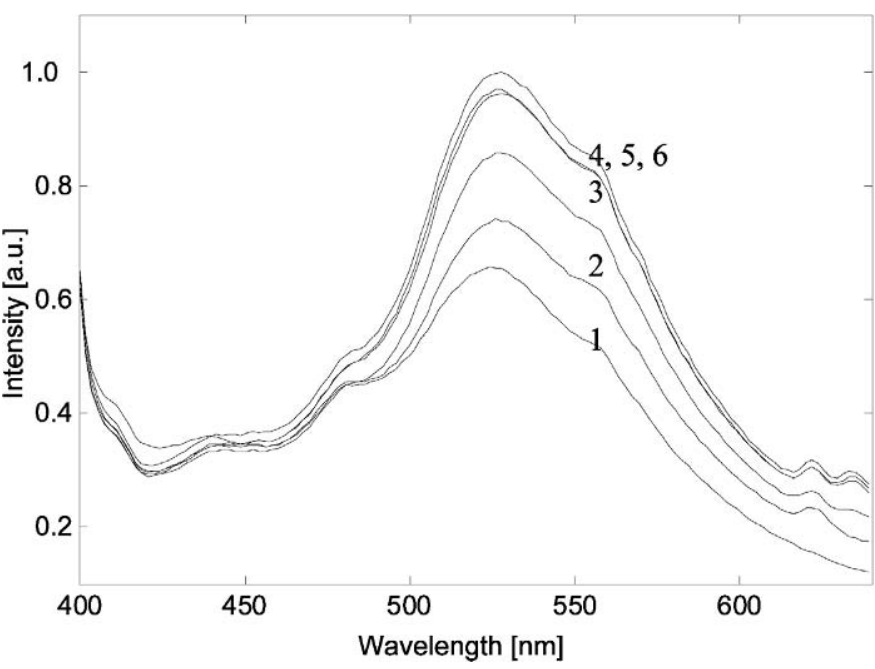

Figure 10. Fluorescence spectra from 7-d illuminated cream cheese. The numbers indicate layer number from surface of cheese (Figure 1).

1). The correlations are high, especially taking into consideration the general uncertainty of sensory analysis. Even though the number of samples was low, the result strongly indicate a close correlation between the fluorescence spectra and the sensory properties of the cheese. Yet, it is not clear whether the correlation is attributed to fluorescence caused by the reduction of riboflavin, formation of lipid oxidation products, the small peaks at 620 to $630 \mathrm{~nm}$, or a combination of these. The data basis is inadequate to draw any clear conclusions, but a careful analysis of the regression components suggests that the region 620 to $630 \mathrm{~nm}$ was very informative, as well as the broad peak at $525 \mathrm{~nm}$. This finding urges the elucidation of the emission peaks at 620 and $630 \mathrm{~nm}$.

\section{General Discussion}

We have observed four separate phenomena in these experiments:

A significant reduction in fluorescence intensity at approximately $525 \mathrm{~nm}$, and a corresponding increase in the region 415 to $490 \mathrm{~nm}$, as a result of illumination. This effect applied to all the products tested and can probably be ascribed to photodegradation of riboflavin.

Variation in two smaller peaks at approximately 620 and $630 \mathrm{~nm}$ seems to be an interaction effect between exposure to light and air.

A very pronounced interaction effect between light and air producing intense blue fluorescence and offflavors on Jarlsberg cheese.

A close correlation between fluorescence spectra and sensory measured off-flavors. 
Table 1. Partial least squares regression (PLSR) results for fluorescence with respect to sensory properties measured inwards in the cheese.

\begin{tabular}{llll}
\hline & No. PLSR & & \\
& components & $\mathrm{R}$ & RMSECV $^{1}$ \\
\hline Oxidized odor & 3 & 0.93 & 0.76 \\
Oxidized flavor & 4 & 0.87 & 1.18 \\
Total odor & 1 & 0.92 & 0.22 \\
Total flavor & 4 & 0.83 & 0.61 \\
\hline
\end{tabular}

${ }^{1} \mathrm{RMSECV}=$ Root mean square error of cross validation.

It was also shown that simple image analysis can reveal how far into the product the light-induced oxidation propagates. With this technique one can also effectively visualize and study the kinetics of this process in different products.

The present study has revealed some interesting properties of solid sample fluorescence from dairy products. The consistent results from the different products together with high correlations with sensory analysis make this detection method very promising. Methods to measure light-induced oxidation in dairy products have been sought, but currently no satisfactory techniques are available (Kristensen et al., 2000). This method might fulfill some of the needs in that respect.

The experiments also leave several unanswered questions. To verify the hypothesis that we are indeed measuring the degree of light-induced degradation of riboflavin careful reference analyses should be performed. If verified, the method could probably be used for rapid quantification of riboflavin content in foods. The origin and impact of the peaks at 620 to $630 \mathrm{~nm}$ should be elucidated. The sensitivity of fluorescence with regard to time/intensity of light and oxygen exposure, as well as to sensory properties should also be investigated. Preliminary measurements at our lab (not yet published) suggest that cheese under normal light exposure after $1 \mathrm{~d}$ of storage can be distinguished from cheese stored in the dark.

The novel use of solid sample fluorescence spectroscopy should certainly be explored further, since the method is rapid, requires no sample preparation, and is easily automated. Finally, we note that the optical measurements performed in this study were technically not very advanced. Therefore, it should be easy to implement a dedicated, simpler system to reduce cost.

\section{CONCLUSIONS}

Based on results of the present work as well as the previous broad study of photodegradation of riboflavin and light-induced lipid oxidation in milk, we propose that:
Solid-sample fluorescence can be used as a nondestructive and rapid tool to measure the degree of lightinduced degradation of riboflavin in dairy products.

Images of fluorescence can be used to visualize the intensity and propagation of this process.

Solid-sample fluorescence can be used as a nondestructive and rapid tool to measure sensory properties connected to storage of dairy products.

Since light catalyzed degradation of riboflavin is a precursor for off-flavors, the method might be capable of detecting early oxidation in dairy products.

The simplicity and rapidity of the method offer rich opportunities for efficient evaluation of factors affecting light-induced oxidation in dairy products, such as packaging materials, light sources, exposure time, and temperature.

\section{ACNOWLEDGMENTS}

We would like to thank TINE for funding the study and providing samples. We are grateful to Kjell Nilsen for valuable discussions, the sensory panel at TINE and to Inger Johanne Fjøsne for her skilled and enthusiastic assistance at the sensory kitchen at MATFORSK. We would also like to thank Elisabeth McGrath, CPAC, for her editing help.

\section{REFERENCES}

Allen, C., and O. W. Parks. 1979. Photodegradation of riboflavin in milks exposed to fluorescent light. J. Dairy Sci. 62:1377-1379.

Aubourg, S. P. 1999. Recent advances in assessment of marine lipid oxidation by using fluorescence. JAOCS 76:409-419.

Aurand, L. W., N. H. Boone, and G. G. Giddings. 1976. Superoxide and singlet oxygen in milk lipid peroxidation. J. Dairy Sci. 60:363-369.

Bekbölet, M. 1990. Light effects on food. J. Food Prot. 53:430-440.

Deger, D., and S. H. Ashoor. 1987. Light-induced changes in taste, appearance, odour, and riboflavin content of cheese. J. Dairy Sci. 70:1371-1376.

Fox, J. B., and D. W. Thayer. 1998. Radical oxidation of riboflavin. Int. J. Vit. Nutr. Res. 68:174-180.

Hansen, E., and L. H. Skibsted. 2000. Light-induced oxidative changes in a model dairy spread. Wavelength dependence of quantum yields. J. Agric. Food Chem. 48:3090-3094.

Hasegawa, K., Y. Endo, and K. Fujimoto. 1992. Oxidative deterioration in dried fish model systems assessed by solid sample fluorescence spectrophotometry. J. Food Sci. 57:1123-1126. 
Hong, C. M., W. L. Wendorff, and J. R. Bradley. 1995. Effects of packaging and lighting on pink discoloration and lipid oxidation of anatto-colored cheeses. J. Dairy Sci. 78:1896-1902.

ISO. 1985a. Sensory Analysis-Methodology-Flavour profile methods. 6564. International Organization for Standardization: Geneva.

Kikugawa, K., and M. Beppu. 1987. Involvement of lipid oxidation products in the formation of fluorescent and cross-linked proteins. Chem. Physics Lipids 44:277-296.

Kristensen, D., and L. H. Skibsted. 1999. Comparison of three methods based on electron spin resonance spectrometry for evaluation of oxidative stability of processed cheese. J. Agric. Food Chem. 47:3099-3104.

Kristensen, D., V. Orlien, G. Mortensen, P. Brockhoff, and L. H. Skibsted. 2000. Light-induced oxidation in sliced havarti cheese packaged in modified atmosphere. Int. Dairy J. 10:95-103.

Li, T. L., J. M. King, and D. B. Min. 2000. Carotenoids in roboflavin photosensitized singlet oxygen oxidation of vitamin $\mathrm{D}_{2}$. J. Food Biochem. 24:477-492.
Liang, J. H. 1999. Use of intrinsic fluorescence in the measurement of oxidative deterioration in milk powders. Food Science Agric. Chem. 1:250-256.

Martens, H., and T. Næs. 1989. Multivariate Calibration. John Wiley and Sons, Chichester, UK.

Parks, O. W., and C. Allen. 1977. Photodegradation of riboflavin to lumichrome in milk exposed to sunlight. J. Dairy Sci. 60:1038.

Toyosaki, T., and A. Hayashi. 1993. Structural analysis of the products of milk riboflavin photolysis. Milchwissenshaft-Milk Sci. Int. 48:607-609.

Wold, J. P., and K. Kvaal. 2000. Mapping lipid oxidation in chicken meat by multispectral imaging of autofluorescence. Appl. Spectr. $54: 900-909$

Wold, J. P., and M. Mielnik. 2000. Non-destructive assessment of lipid oxidation in minced poultry meat by autofluorescence spectroscopy. J. Food Sci. 65:87-95.

Woodcock, E. A., J. J. Warthesen, and T. P. Labuza. 1982. Riboflavin photochemical degradation in pasta measured by high performance liquid chromatography. J. Food Sci. 47:545-549. 